\title{
Genome-Wide Analysis of HAK/KUP/KT Potassium Transporter Genes in Banana (Musa Acuminata L.) and Their Tissue-Specific Expression Profiles Under Potassium Stress
}

\section{Siwen Liu ( $\square$ wenwen901203@hotmail.com )}

Guangdong Academy of Agricultural Sciences https://orcid.org/0000-0002-0446-0448

\section{Bangting Wu}

Guangdong Academy of Agricultural Sciences

\section{Yanling Xie}

Guangdong Academy of Agricultural Sciences

\section{Sijun Zheng}

Yunnan Academy of Agricultural Sciences

Jianghui Xie

Chinese Academy of Tropical Agricultural Sciences

\section{Wei Wang}

Chinese Academy of Tropical Agricultural Sciences

\section{Dandan Xiang}

Guangdong Academy of Agricultural Sciences

\section{Chunyu Li}

Guangdong Academy of Agricultural Sciences

\section{Research Article}

Keywords: Banana , Potassium , HAK/KUP/KT gene family , abiotic stress, expression profiles

Posted Date: August 3rd, 2021

DOl: https://doi.org/10.21203/rs.3.rs-745958/v1

License: (c) (i) This work is licensed under a Creative Commons Attribution 4.0 International License. Read Full License

Version of Record: A version of this preprint was published at Plant Growth Regulation on February 11th, 2022. See the published version at https://doi.org/10.1007/s10725-021-00793-7. 


\section{Abstract}

Potassium is one of the most essential inorganic cations for plant growth and development. The high affinity $\mathrm{K}^{+}(\mathrm{HAK}) / \mathrm{K}^{+}$uptake (KUP)/ $\mathrm{K}^{+}$transporter $(\mathrm{KT})$ family plays essential roles in the regulation of cellular $\mathrm{K}^{+}$levels and the maintenance of osmotic balance. However, the roles of these genes in the responses of bananas to low-potassium stress are unclear. In this study, 24 HAK/KUP/KT (MaHAK) genes were identified from banana genomic data. These genes were further classified into four groups based on phylogenetic analysis, gene structure and conserved domain analysis. Segmental duplication events played an important role in the expansion of the MaHAK gene family. Transcriptome analysis revealed the expression patterns of MaHAKs in various tissues under different $\mathrm{K}^{+}$conditions. MaHAK14b was upregulated under both short- and long-term $\mathrm{K}^{+}$-deficient conditions, suggesting that it plays crucial roles in $\mathrm{K}^{+}$uptake at low $\mathrm{K}^{+}$concentrations. Furthermore, MaHAK14b mediated $\mathrm{K}^{+}$uptake when it was heterologously expressed in the yeast mutant R5421 on low $\mathrm{K}^{+}$medium. Collectively, these findings provide a foundation for further functional analysis of MaHAK genes, which may be used to improve stress resistance in bananas.

\section{Introduction}

Potassium ions $\left(\mathrm{K}^{+}\right)$are one of the most essential plant macronutrients and are involved in various plant physiological and biochemical processes, such as osmoregulation, enzyme activation, stomatal regulation, and maintenance of $\mathrm{pH}$ (Hasanuzzaman et al. 2018). Plants are often subjected to potassium deficiency, which adversely impacts plant growth by influencing photosynthesis, carbohydrate metabolism and transpiration rates (Pettigrew 2008). Potassium uptake by plant roots and its translocation inside plants rely on a complex transport system consisting of numerous transporters and channels (Epstein and Kim 1971). Potassium transporters in plants can be classified into four families: the high affinity $\mathrm{K}^{+}(\mathrm{HAK}) / \mathrm{K}^{+}$uptake (KUP)/ $\mathrm{K}^{+}$transporter (KT) family, the Trk/Ktr/HKT family, the $\mathrm{K}^{+}$ efflux antiporter (KEA) family, and the cation/ $\mathrm{H}^{+}$exchanger (CHX) family (Maser et al. 2001; Gierth and Maser 2007).

HAK/KUP/KT is the largest potassium transporter family and has been further divided into four groups:

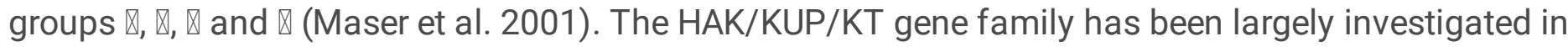
many plants, e.g., Arabidopsis thaliana (Maser et al. 2001), Oryza sativa (Gupta et al. 2008), Zea mays (Zhang et al. 2012), Prunus persica (Song et al. 2015), Cicer arietinum (Azeem et al. 2018), Manihot esculenta (Ou et al. 2018), and Triticum aestivum (Cheng et al. 2018). The number of HAK genes varies among species, ranging from 13 to 56, with the lowest number in $A$. thaliana and the highest number in $T$. aestivum (Maser et al. 2001; Cheng et al. 2018). Expression analysis of HAK/KUP/KT genes from a variety of species with different tissues suggests the functional diversity of these genes. In Arabidopsis, most AtKT/KUP/HAK genes are expressed in the root, siliques, leaves and flowers (Ahn et al. 2004). In rice, five genes were shown to be expressed in all tissues (Gupta et al. 2008). In wheat, most genes from groups $\nabla$ and $\nabla$ were strongly expressed in all tissues (Cheng et al. 2018). In addition, the expression 
profiles of HAK/KUP/KT genes in response to different abiotic stresses have also been investigated in many plant species (Cheng et al. 2018).

Generally, the main functions of HAK/KUP/KT transporters are to regulate plant growth and development and to conduct high-affinity $\mathrm{K}^{+}$uptake to maintain cellular $\mathrm{K}^{+}$homeostasis under low $\mathrm{K}^{+}$conditions (Wang and Wu 2013; Li et al. 2018). In Arabidopsis, AtKUP4 is related to cell expansion of root hairs (Rigas et al. 2001). AtHAK5 is the most important transporter involved in $\mathrm{K}^{+}$uptake in roots, and it is highly upregulated after $\mathrm{K}^{+}$starvation (Pyo et al. 2010). In addition, AtKUP7 is also confirmed to be involved in $\mathrm{K}^{+}$uptake and upregulated under $\mathrm{K}^{+}$-deficient conditions (Han et al. 2016). In rice, OsHAK1 and OsHAK5 are functionally characterized. Overexpressing OsHAK5 or OsHAK1 in rice increased $\mathrm{K}^{+}$ uptake under $\mathrm{K}^{+}$-limited conditions, and they are all mainly expressed in roots, where they mediate $\mathrm{K}^{+}$ uptake and $\mathrm{K}^{+}$translocation (Yang et al. 2014; Chen et al. 2015; Chen et al. 2017). ZmHAK5 shares a similar function with AtHAK5 and plays a role in $\mathrm{K}^{+}$uptake in maize roots (Qin et al. 2019). Loss of function of ZmHAK5 led to defective growth and $\mathrm{K}^{+}$uptake under low $\mathrm{K}^{+}$conditions. $\mathrm{ZmHAK} 1$ has also been confirmed as a high-affinity $\mathrm{K}^{+}$transporter, but its activity is weaker than that of ZamHAK5 (Qin et al. 2019).

Banana (Musa acuminata L.) is one of the most important fruits and a staple crop in the world with high economic value. Compared to most of the other crops, banana plants have a very high demand for potassium. The yield and fruit quality of banana are directly related to $\mathrm{K}^{+}$availability; therefore, a large amount of $\mathrm{K}^{+}$fertilizer is usually applied in banana-planting fields (Rufyikiri et al. 2003). Characterization of HAK/KUP/KT family members would be helpful for improving $\mathrm{K}^{+}$utilization efficiency in banana. In this study, we identified $24 \mathrm{HAK} / \mathrm{KUP} / \mathrm{KT}$ transporters from the genome of banana and analyzed their phylogenetic relationships, conserved motifs, gene structures, and expression profiles in response to different $\mathrm{K}^{+}$stresses using our transcriptome data and publicly available data. In addition, we cloned the MaHAK14b gene, which was highly upregulated in $\mathrm{K}^{+}$-deficient conditions, and demonstrated its roles in facilitating $\mathrm{K}^{+}$transport in yeast.

\section{Materials And Methods}

\section{Identification of HAK/HUP/KT genes in banana}

The hidden Markov model (HMM) profile of the HAK/HUP/KT transporter family (Pfam ID: PF02705) was obtained from the Pfam protein family database (http://pfam.xfam.org/). HMMER 3.3 was used to search the HAK/HUP/KT genes from the banana genome database, which were downloaded from Banana Genome Hub (https://banana-genome-hub.southgreen.fr/). Proteins with E-value $<0.01$ was obtained. Then, all the candidate MaHAK protein sequences were further submitted to CDD databases and SMART to verify the presence of the $\mathrm{K}+$ transporter domain. 


\section{Phylogenetic analysis of MaHAKs}

All the HAK/KUP/KT gene sequences of $A$. thaliana, $O$. sativa and $Z$. mays were retrieved from NCBI (https://www.ncbi.nlm.nih.gov/). Multiple alignment of all HAKs of different plant species was carried out using ClustalW, and the phylogenetic tree was drawn using the neighbor-joining ( $N J$ ) method of MEGA v. 7.0, tested by 1000 Bootstrap replicated (Kumar et al. 2016).

\section{Structural analysis of MaHAKs}

The intron and exon distribution patterns of each MaHAK gene were illustrated using Gene Structure Display Server v. 2.0 (Hu et al. 2015). The MEME online program (http://meme.nbcr.net/meme/intro.html) was used to identify conserved motifs in MaHAK proteins. Conserved motifs of the MaHAKs proteins.

\section{Chromosome localization and syntenic relationship analysis}

MaHAK genes were mapped to banana chromosomes based on physical location information from the banana genome database using Circos (Krzywinski et al. 2009). The gene duplication events were analyzed by the Multiple Collinearity Scan toolkit (MCScanX), and the syntenic analysis map was constructed using Tbtools (Chen et al. 2020).

\section{Plant growth condition}

"Cavendish" banana (AAA) cv "Brazilian" plantlets with six to seven leaves (c. $25 \mathrm{~cm}$ height) were cultured in a greenhouse at a temperature of $28-30^{\circ} \mathrm{C}$. The plants were first cultured in water for $1 \mathrm{~d}$, divided into normal and low-K+ groups, and cultured in full nutrient MS medium and potassium-free MS medium for 4 $h$, respectively. Then, the roots, pseudostems and leaves of each sample were collected.

\section{Transcriptome analysis}

Total RNA was extracted from each sample using an Illumina standard library preparation kit. Each treatment had three biological replicates. RNA-seq reads were obtained by Illumina paired-end sequencing on the Illumina HiSeq2500 platform. Paired-end clean reads were mapped to the banana reference genome (Musa acuminate DH Pahang v2) using HISAT2.2.4 with "-rna-strandness RF. Fragments per kilobase of transcript per million mapped reads (FPKM) were used to calculate gene expression levels.

We further analyzed RNA-seq data of long-term potassium stress in banana roots from a publicly available database (PRJNA399649). The expression levels of the MaHAK genes were normalized and represented as FPKM values, which were used to generate a heatmap with Tbtools (Chen et al. 2020). 


\section{Subcellular location of MaHAK14b}

The coding sequence of MaHAK14b was amplified and inserted into the pCAMBIA1300:GFP vector. The vector harboring GFP, MaHAK14b-GFP, and plasma membrane markers was transferred into Agrobacterium tumefaciens. Then, the Nicotiana benthamiana leaves were filtered with A. tumefaciens cells. The leaves were analyzed for fluorescence $2 \mathrm{~d}$ after transformation using a confocal laser scanning microscope (LSM 710; Carl Zeiss, Oberkochen, Germany).

\section{Yeast complementation}

The coding sequence of MaHAK14b was cloned into the pYES2 vector. pYES2-MaHAK14b and empty vector were transformed into the potassium uptake-deficient yeast strain R5421 (trk1 $\Delta$, trk2 $\Delta$ ). The yeast transformants were grown in YPDA medium supplemented with $100 \mathrm{mM} \mathrm{KCl}$ at $30^{\circ} \mathrm{C}$ until the $\mathrm{OD}_{600}$ reached 0.8. Cells were harvested by centrifugation at $5,000 \mathrm{~g}$ for $1 \mathrm{~min}$, washed twice with sterile water and then resuspended in sterile water to an $\mathrm{OD}_{600}$ of 0.8 . The yeast cells were diluted 10 -fold, 100 -fold, and 1000 -fold and cultured in AP plates containing $1 \mathrm{mM}, 10 \mathrm{mM}$ and $100 \mathrm{mM} \mathrm{KCl}$. The plates were cultured at $30^{\circ} \mathrm{C}$ for $3 \mathrm{~d}$ before taking pictures.

\section{Results}

\section{Identification of the MaHAKs in banana}

A total of 26 HAK genes were originally obtained from the banana A genome using the HMM search. Two erroneously predicted genes (Ma02_04550, Ma07_18090) were manually removed after confirming the presence of the $\mathrm{K}^{+}$transporter domain. Finally, 24 genes were identified as banana HAK genes and were renamed according to their corresponding homologs from rice and maize based on phylogenetic relationship analysis. Fourteen family members and the letters $a, b, c$ and $d$ were added when more than one gene clustered together with the specific rice gene, for instance, MaHAK10a, MaHAK10b, MaHAK10c and MaHAK10d (Table 1). The composition of HAK members in the banana genome was very different from that in rice, maize or Arabidopsis. No MaHAK genes homologous to OsHAK3, OsHAK4, OsHAK6, OsHAK15, OsHAK16, OsHAK19, OsHAK20, OsHAK21, OsHAK22, OsHAK24, OsHAK25, OsHAK26 or OsHAK27 were identified. However, seven MaHAKs had two or three or even four homologs, including MaHAK2, MaHAK7, MaHAK8, MaHAK10, MaHAK11, MaHAK13 and MaHAK14. The length of MaHAK genes ranged from 565 to 839 amino acids (aa). Each MaHAK protein contained at least 8 transmembrane segments. The molecular weight of the MaHAK proteins ranged from 63.54 to $93.82 \mathrm{kDa}$.

Table 1

The HAK/HUP/KT genes in Musa acuminata L. 


\begin{tabular}{|c|c|c|c|c|}
\hline Gene Name & Gene ID & Amino Acid Length & TMs & $\mathrm{MW}(\mathrm{kDa})$ \\
\hline MaHAK1 & Ma05_g07130 & 633 & 11 & 70.02 \\
\hline MaHAK2a & Ma11_g18830 & 785 & 13 & 87.66 \\
\hline MaHAK2b & Ma08_g09830 & 638 & 8 & 71.27 \\
\hline MaHAK5 & Ma10_g17110 & 752 & 12 & 83.28 \\
\hline MaHAK7a & Ma10_g01080 & 782 & 13 & 88.14 \\
\hline MaHAK7b & Ma05_g07770 & 781 & 13 & 87.76 \\
\hline MaHAK8a & Ma11_g23250 & 769 & 13 & 86.12 \\
\hline MaHAK8b & Ma08_g01620 & 779 & 11 & 86.39 \\
\hline MaHAK9 & Ma07_g23610 & 771 & 11 & 85.62 \\
\hline MaHAK10a & Ma09_g23160 & 774 & 11 & 85.90 \\
\hline MaHAK10b & Ma01_g00350 & 781 & 10 & 86.43 \\
\hline MaHAK10c & Ma07_g21520 & 771 & 11 & 86.31 \\
\hline MaHAK10d & Ma03_g06090 & 782 & 13 & 87.28 \\
\hline MaHAK11a & Ma05_g00190 & 789 & 14 & 88.16 \\
\hline MaHAK11b & Ma08_g33820 & 794 & 14 & 88.38 \\
\hline MaHAK11c & Ma11_g16690 & 780 & 14 & 87.64 \\
\hline MaHAK12 & Ma02_g04540 & 565 & 11 & 63.54 \\
\hline MaHAK13a & Ma08_g06810 & 736 & 12 & 81.47 \\
\hline MaHAK13b & Ma03_g24780 & 740 & 11 & 81.88 \\
\hline MaHAK14a & Ma08_g19550 & 836 & 10 & 93.82 \\
\hline MaHAK14b & Ma09_g11150 & 839 & 10 & 93.66 \\
\hline MaHAK17 & Ma07_g12190 & 704 & 9 & 78.10 \\
\hline MaHAK18 & Ma04_g39690 & 774 & 13 & 86.34 \\
\hline MaHAK23 & Ma07_g21540 & 829 & 13 & 92.70 \\
\hline
\end{tabular}

\section{Phylogenetic analysis of MaHAKs in banana}

The evolutionary relationship of HAKs in banana, rice, maize and Arabidopsis was analyzed by constructing a phylogenetic tree (Figure 1). Based on previous studies of HAK proteins in Arabidopsis and 


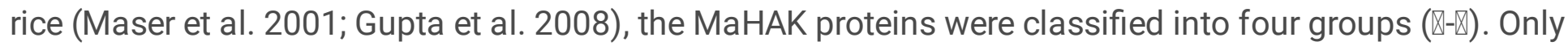
MaHAK5 was placed in Group \&; MaHAK1, 2, 7, 8, 9, 10, 13 were placed in Group \&; MaHAK11, 12, 14, 18

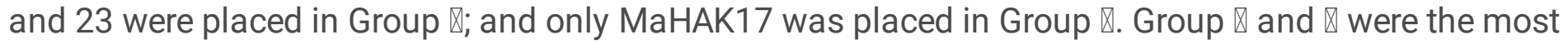
abundant in banana, comprising $54 \%$ and $40 \%$ proteins of all MaHAKs, respectively.

\section{Gene structure and conserved motif examination of MaHAKs in banana}

To further gain more insight into the evolution of the HAK family in banana, the exon-intron organizations of all identified MaHAK genes were examined. As shown in Figure 2, the coding sequences of all MaHAK genes were disrupted by introns, with numbers ranging from 5 to 10 . Homologous genes usually have similar structures; for example, the three MaHAK11 genes contained 9 exons and 8 introns.

To assess the conservation and diversification of MaHAK proteins, protein motifs were further analyzed using the online program MEME. Motifs 1, 2, 3, 4, 6, 9 and 10 were conserved in all MaHAK proteins. It was also obvious that the motif composition was similar among homologous genes. Interestingly, the lengths of introns between the two homologous genes MaHAK7a and MaHAK7b were quite different, while the motif distributions were highly similar.

\section{Genomic distribution and evolutionary analysis of MaHAKs in banana}

MaHAK genes were unevenly distributed in 10 of 11 banana chromosomes (chr), except chr06 (Figure 3). Overall, the MaHAK gene was likely to be located in the distal parts of the chromosomes. Chr08 contained the largest number of MaHAK genes (MaHAK2b, MaHAK8b, MaHAK11b, MaHAK13a, MaHAK14a). To further investigate the duplication event occurring in the MaHAK genes, a synteny analysis was conducted. Six pairs of syntenic genes, MaHAK2a (chr11)-MaHAK2b (chr08), MaHAK8b (chr08)-MaHAK9 (chr07), MaHAK10c (chr07)-MaHAK10d (chr03), MaHAK11a (chr05)-MaHAK11c (chr11), MaHAK13a (chr08)-MaHAK13b (chr03) and MaHAK14a (chr08)-MaHAK14b (chr09), were found in MaHAKs (Figure 4). These results indicated that gene duplication events play an important driving force for MaHAK evolution.

\section{Expression profile of MaHAKs under different potassium treatments}

To study the expression profiles of MaHAK genes in different tissues under different potassium stresses, transcriptome analyses of the roots, pseudostems and leaves in the Baxi cultivar under normal $\left(+\mathrm{K}^{+}\right)$and potassium- deficient $\left(-\mathrm{K}^{+}\right)$conditions were performed. As shown in Figure $5 \mathrm{a}$, most MaHAK genes 
exhibited moderate expression in different tissues under the different treatments, and the expression of MaHAK13a was very low or undetectable. MaHAK1, 2b and 10d showed low expression levels in roots, and MaHAK17 was expressed at low levels in both roots and pseudostems under both $\mathrm{K}^{+}$treatment conditions. MaHAK14b showed very low expression in roots under normal $\mathrm{K}^{+}$conditions but was upregulated under $\mathrm{K}^{+}$-deficient conditions. In addition, MaHAK14b was normally expressed at low levels in leaves in the $\mathrm{K}^{+}$treatment conditions but was highly expressed in the pseudostem. MaHAK7b was indeed upregulated under $\mathrm{K}^{+}$-deficient treatment in roots and leaves. To analyze the expression pattern of MaHAK genes when symptoms appeared (60 d after treatment) under potassium stress, FPKM (kilobase/million mapped reads) data of MaHAKs were extracted (Xu et al. 2019). Six MaHAK genes were differentially expressed in normal- $K^{+}$roots (NKRs) versus low- $K^{+}$roots (LKRs) (Figure $5 b$ ). MaHAK10c and MaHAK10d were strongly upregulated and downregulated after low- $\mathrm{K}^{+}$treatment, respectively. These results implied the different roles of these MaHAK genes in different tissues under different $\mathrm{K}^{+}$conditions.

\section{Functional characterization of MaHAK1 in potassium uptake}

According to the increased expression of MaHAK14b induced by $\mathrm{K}^{+}$deficiency in banana roots, MaHAK14b might be a high-affinity $\mathrm{K}^{+}$uptake transporter from banana. First, the subcellular location of MaHAK14b was examined by generating a construct expressing a fusion protein of MaHAK14b-GFP (green fluorescent protein). The recombinant construct and control vector were introduced into the leaves of Nicotiana benthamiana. As shown in Figure 6a, the results indicate that MaHAK14b was solely located in the plasma membrane, while the green fluorescence of the GFP control was located in the nucleus, cytoplasm and plasma membrane.

Several reported HAK proteins could complement the growth defect of potassium-deficient yeast strain R5421, e.g., OsHAK1, OsHAK5 and ZmHAK5 (Yang et al. 2014; Chen et al. 2015; Qin et al. 2019). To examine the potassium uptake activity of MaHAK14b, R5421 yeast were transformed with MaHAK14b and the vector as a control. The growth of transformants was compared on AP medium containing various $\mathrm{K}^{+}$concentrations. Yeast R5421 expressing MaHAK14b grew in $1 \mathrm{mM} \mathrm{KCl}$, while no growth was observed in R5421 transformed with empty vector (Figure 6b). This result indicates that MaHAK14b is a high-affinity $\mathrm{K}^{+}$transporter with $\mathrm{K}^{+}$uptake activity.

\section{Discussion}

The HAK/KUP/KT gene family is the largest $\mathrm{K}^{+}$transporter family and plays important roles in $\mathrm{K}^{+}$ transport across membranes in bacteria, fungi, and plants (Li et al. 2018). Currently, the HAK family has been reported in several plant species, such as Arabidopsis, rice, maize, wheat, cassava and peach (Maser et al. 2001; Gupta et al. 2008; Zhang et al. 2012; Song et al. 2015; Cheng et al. 2018; Ou et al. 
2018). However, a systematic analysis of the HAK gene family in banana has not yet been conducted. In this study, we identified 24 HAK genes in the banana genome, which were distributed into 4 groups based on their evolutionary relationships with Arabidopsis, rice and maize. The classification of MaHAKs was also supported by gene structure and conserved motif analyses. The exon number of MaHAK genes ranged from 6 to 11, which was different from that of rice (2 to 10) and maize (3 to 10) (Gupta et al. 2008; Zhang et al. 2012) and similar to that of peach (7-12) and cassava (6-10) (Song et al. 2015; Ou et al. 2018). The reason for the difference may lie in the absence of homologs of HAK4 (5 exons) and HAK22 (3 exons) in banana.

The HAK gene number of banana is higher than that of Arabidopsis (13 members) and relatively lower than that of rice (27 members) and maize (27 members) (Gupta et al. 2008; Zhang et al. 2012). However, the gene diversification of MaHAKs was lower than that of OsHAKs and ZmHAKs, with only 14 kinds of HAK genes. The high number of members of MaHAKs indicates an event of succession of expansion, and extensive duplication occurred in the banana genome. In particular, MaHAK10 and MaHAK11 are significantly expanded in banana compared with other species, while banana orthologs of 13 OsHAKs of rice could not be identified. These results indicate that there might be gene loss in the lineage leading to Musa and gene expansion within Musa acuminata L.

Gene duplication events are essential for the expansion and evolution of gene families (Maher et al.

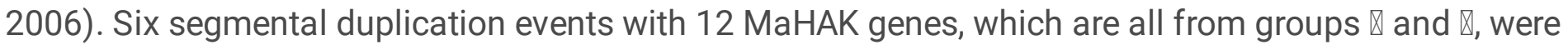
identified with MCScanX methods. Remarkably, these genes tend to be located in distal telomeric segments of chromosomes, which are evolutionary hotspots consisting of many fast-evolving genes

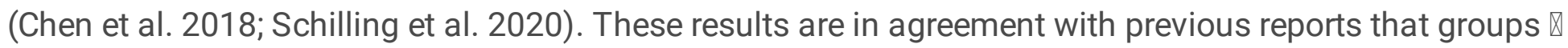
and $\nabla$ of HAK/KUP/KTs have substantial functional diversity (Yang et al. 2009). Further studies are required to characterize the functions of these duplicated genes in the adaptability of banana to different $\mathrm{K}^{+}$conditions.

To further understand the functions of MaHAK genes, their expression profiles were analyzed in various banana tissues under different $\mathrm{K}^{+}$conditions. Generally, $\mathrm{K}^{+}$transporter genes are upregulated under low$\mathrm{K}^{+}$conditions. In Arabidopsis, AtHAK5 was strongly induced by low- $\mathrm{K}^{+}$stress and was the most important AtKT/KUP/HAK involved in $\mathrm{K}^{+}$uptake in roots (Ahn et al. 2004; Pyo et al. 2010). OsHAK1 and OsHAK5 in rice were upregulated under $\mathrm{K}^{+}$-deficient conditions, with increased $\mathrm{K}^{+}$uptake in rice roots (Yang et al. 2014; Chen et al. 2015; Chen et al. 2017). We found that the expression pattern of many MaHAK genes is different from that of close homologs in other species. MaHAK5 did not display a similar expression pattern in banana roots. In both the short- and long-term low- $\mathrm{K}^{+}$treatments, the transcript levels of MaHAK1 and MaHAK5 did not change significantly in banana roots, whereas that of MaHAK5 increased in leaves. These findings suggest that MaHAK transcription is regulated by a different mechanism than homologs in other plants, or perhaps the expression pattern of MaHAKs varies during different periods of low $\mathrm{K}^{+}$conditions. 
Additionally, we found that MaHAK14b was rapidly induced after $4 \mathrm{~h}$ of $\mathrm{K}^{+}$deficiency and that none was upregulated after $60 \mathrm{~d}$ of $\mathrm{K}^{+}$starvation in banana roots. A yeast complementation assay was conducted to further demonstrate that MaHAK14b functions in $\mathrm{K}^{+}$uptake at a $1 \mathrm{mM} \mathrm{K}^{+}$concentration. These results provide insight into the potential roles of MaHAK14b in $\mathrm{K}^{+}$uptake under low $\mathrm{K}^{+}$conditions in banana roots.

\section{Conclusion}

In summary, a total of 24 MaHAK genes were identified from the banana genome. The MaHAK gene family was comprehensively analyzed by assessing phylogenetic relationships, gene structures, chromosomal distribution, synteny and expression patterns. The present study provides fundamental knowledge for further studying the functional mechanism of potassium transporters in banana development and abiotic stress responses.

\section{Declarations}

\section{Funding}

This research was financially supported by the Guangdong Basic and Applied Basic Research Foundation (2020A1515110731), the Director Fund of the Institute of Fruit Research, Guangdong Academy of Agricultural Sciences, the Special Fund for Scientific Innovation Strategy-Construction of High Level Academy of Agriculture Science (R2018QD-021), the Special Fund Project for Talent Introduction of Guangdong Academy of Agriculture Science.

\section{Conflict of interest}

The authors declare that they have no known competing interests.

\section{References}

1. Ahn SJ, Shin R and Schachtman DP (2004) Expression of KT/KUP genes in Arabidopsis and the role of root hairs in $\mathrm{K}^{+}$uptake. Plant Physiol 134(3), 1135-45

2. Azeem F, Ahmad B, Atif RM, Ali MA, Nadeem H, Hussain S, Manzoor H, Azeem M and Afzal M (2018) Genome-wide analysis of potassium transport-related genes in Chickpea (Cicer arietinum L.) and their role in abiotic stress responses. Plant Molecular Biology Reporter 36(3), 451-468

3. Chen C, Chen H, Zhang Y, Thomas HR, Frank MH, He Y and Xia R (2020) TBtools: An Integrative Toolkit Developed for Interactive Analyses of Big Biological Data. Mol Plant 13(8), 1194-1202

4. Chen G, Hu Q, Luo L, Yang T, Zhang S, Hu Y, Yu L and Xu G (2015) Rice potassium transporter OsHAK1 is essential for maintaining potassium-mediated growth and functions in salt tolerance over low and high potassium concentration ranges. Plant Cell Environ 38(12), 2747-65 
5. Chen G, Liu C, Gao Z, Zhang Y, Jiang H, Zhu L, Ren D, Yu L, Xu G and Qian Q (2017) OsHAK1, a highaffinity potassium transporter, positively regulates responses to drought stress in rice. Front Plant Sci 8,1885

6. Chen NWG, Thareau V, Ribeiro T, Magdelenat G, Ashfield T, Innes RW, Pedrosa-Harand A and Geffroy $V$ (2018) Common bean subtelomeres are hot spots of recombination and favor resistance gene evolution. Front Plant Sci 9, 1185

7. Cheng X, Liu X, Mao W, Zhang X, Chen S, Zhan K, Bi H and Xu H (2018) Genome-wide identification and analysis of HAK/KUP/KT potassium transporters gene family in wheat (Triticum aestivum L.). Int J Mol Sci 19(12),

8. Epstein W and Kim BS (1971) Potassium transport loci in Escherichia coli K-12. J Bacteriol 108(2), 639-44

9. Gierth $\mathrm{M}$ and Maser $\mathrm{P}$ (2007) Potassium transporters in plants-involvement in $\mathrm{K}^{+}$acquisition, redistribution and homeostasis. FEBS Lett 581(12), 2348-56

10. Gupta M, Qiu X, Wang L, Xie W, Zhang C, Xiong L, Lian X and Zhang Q (2008) KT/HAK/KUP potassium transporters gene family and their whole-life cycle expression profile in rice (Oryza sativa). Mol Genet Genomics 280(5), 437-52

11. Han $\mathrm{M}$, Wu W, Wu WH and Wang $Y(2016)$ Potassium transporter KUP7 is involved in $K\left(^{+}\right)$acquisition and translocation in Arabidopsis root under $\mathrm{K}\left({ }^{+}\right)$-limited conditions. Mol Plant 9(3), 437-446

12. Hasanuzzaman M, Bhuyan M, Nahar K, Hossain M, Mahmud J, Hossen M, Masud A, Moumita and Fujita M (2018) Potassium: A vital Regulator of Plant Responses and Tolerance to Abiotic Stresses. Agronomy 8(3),

13. Hu B, Jin J, Guo AY, Zhang H, Luo J and Gao G (2015) GSDS 2.0: an upgraded gene feature visualization server. Bioinformatics 31(8), 1296-7

14. Krzywinski M, Schein J, Birol I, Connors J, Gascoyne R, Horsman D, Jones SJ and Marra MA (2009) Circos: an information aesthetic for comparative genomics. Genome Res 19(9), 1639-45

15. Kumar S, Stecher G and Tamura K (2016) MEGA7: Molecular Evolutionary Genetics Analysis Version 7.0 for Bigger Datasets. Mol Biol Evol 33(7), 1870-4

16. Li W, Xu G, Alli A and Yu L (2018) Plant HAK/KUP/KT K( $\left.{ }^{+}\right)$transporters: Function and regulation. Semin Cell Dev Biol 74, 133-141

17. Maher $C$, Stein $L$ and Ware $D$ (2006) Evolution of Arabidopsis microRNA families through duplication events. Genome Res 16(4), 510-9

18. Maser P, Thomine S, Schroeder JI, Ward JM, Hirschi K, Sze H, Talke IN, Amtmann A, Maathuis FJ, Sanders D, Harper JF, Tchieu J, Gribskov M, Persans MW, Salt DE, Kim SA and Guerinot ML (2001) Phylogenetic relationships within cation transporter families of Arabidopsis. Plant Physiol 126(4), 1646-67

19. Ou W, Mao X, Huang C, Tie W, Yan Y, Ding Z, Wu C, Xia Z, Wang W, Zhou S, Li K and Hu W (2018) Genome-wide identification and expression analysis of the KUP family under abiotic stress in 
Cassava (Manihot esculenta Crantz). Front Physiol 9, 17

20. Pettigrew WT (2008) Potassium influences on yield and quality production for maize, wheat, soybean and cotton. Physiol Plant 133(4), 670-81

21. Pyo YJ, Gierth M, Schroeder JI and Cho MH (2010) High-affinity $\mathrm{K}\left({ }^{+}\right)$transport in Arabidopsis: AtHAK5 and AKT1 are vital for seedling establishment and postgermination growth under lowpotassium conditions. Plant Physiol 153(2), 863-75

22. Qin YJ, Wu WH and Wang Y (2019) ZmHAK5 and ZmHAK1 function in $K\left(^{+}\right)$uptake and distribution in maize under low $\mathrm{K}\left(^{+}\right)$conditions. J Integr Plant Biol 61(6), 691-705

23. Rigas S, Debrosses G, Haralampidis K, Vicente-Agullo F, Feldmann KA, Grabov A, Dolan L and Hatzopoulos P (2001) TRH1 encodes a potassium transporter required for tip growth in Arabidopsis root hairs. Plant Cell 13(1), 139-51

24. Rufyikiri G, Genon JG, Dufey JE and Delvaux B (2003) Competitive adsorption of hydrogen, calcium, potassium, magnesium, and aluminum on banana roots: experimental data and modeling. Journal of Plant Nutrition 26(2), 351-368

25. Schilling S, Kennedy A, Pan S, Jermiin LS and Melzer R (2020) Genome-wide analysis of MIKC-type MADS-box genes in wheat: pervasive duplications, functional conservation and putative neofunctionalization. New Phytol 225(1), 511-529

26. Song ZZ, Ma RJ and Yu ML (2015) Genome-wide analysis and identification of KT/HAK/KUP potassium transporter gene family in peach (Prunus persica). Genet Mol Res 14(1), 774-87

27. Wang Y and Wu WH (2013) Potassium transport and signaling in higher plants. Annu Rev Plant Biol 64, 451-76

28. Xu M, Zeng C-B, He R, Yan Z, Qi Z, Xiong R, Cheng Y, Wei S-S and Tang H (2019) Transcriptome analysis of banana (Musa acuminate L.) in response to low-potassium stress. Agronomy 9(4),

29. Yang T, Zhang S, Hu Y, Wu F, Hu Q, Chen G, Cai J, Wu T, Moran N, Yu L and Xu G (2014) The role of a potassium transporter OsHAK5 in potassium acquisition and transport from roots to shoots in rice at low potassium supply levels. Plant Physiol 166(2), 945-59

30. Yang Z, Gao Q, Sun C, Li W, Gu S and Xu C (2009) Molecular evolution and functional divergence of HAK potassium transporter gene family in rice (Oryza sativa L.). Journal of Genetics and Genomics 36(3), 161-172

31. Zhang Z, Zhang J, Chen Y, Li R, Wang H and Wei J (2012) Genome-wide analysis and identification of HAK potassium transporter gene family in maize (Zea mays L.). Mol Biol Rep 39(8), 8465-73

\section{Figures}




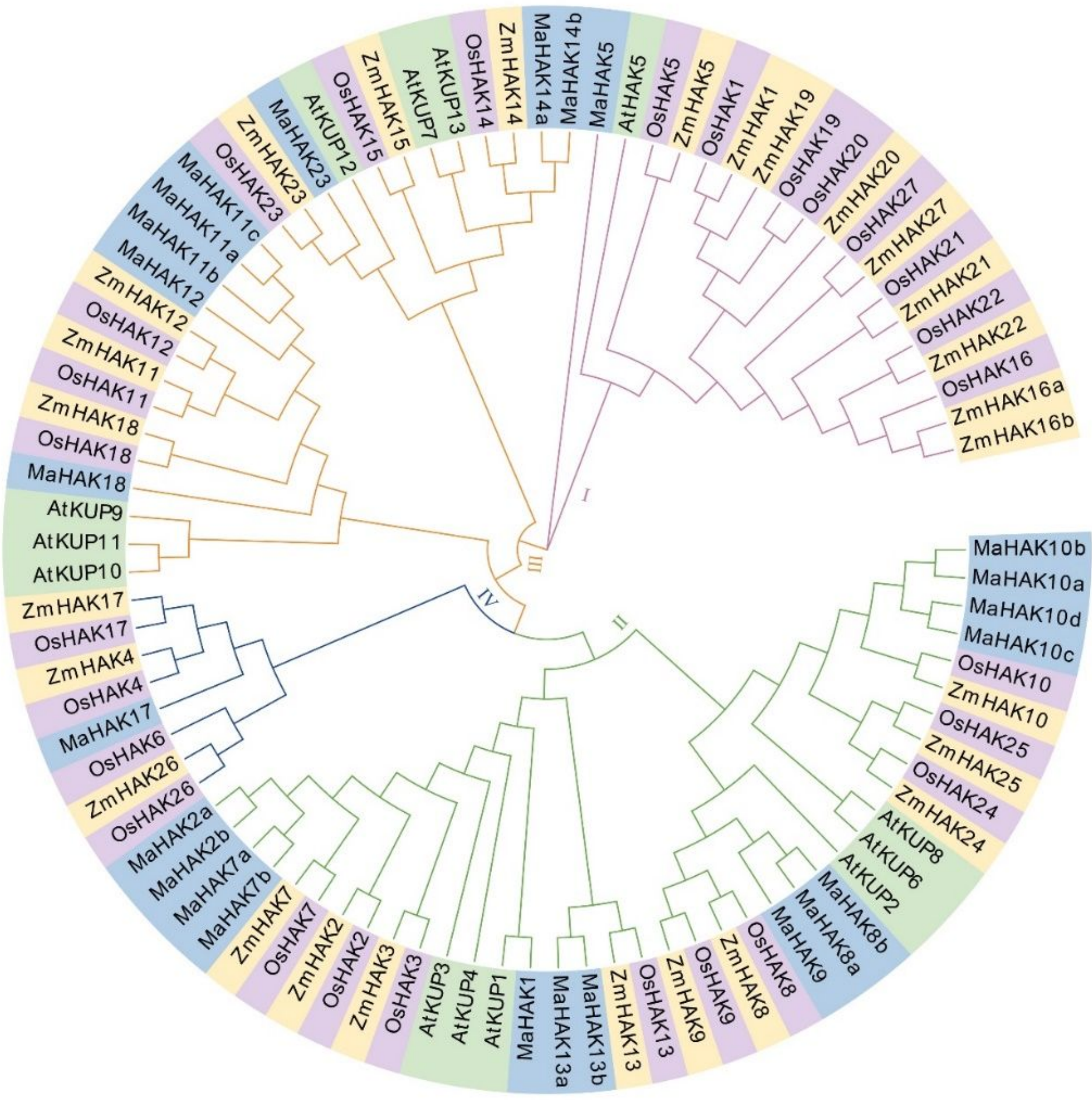

Figure 1

Phylogenetic tree of HAK/KUP/KT (HAK) proteins among different species. The prefixes 'Ma', 'Os', 'Zm', and 'At' indicate HAK proteins from Musa acuminata, Oryza sativa, Zea mays, and Arabidopsis thaliana, respectively. The different-colored lines indicate different groups ( $\mathbb{(}-\mathbb{Z})$ of HAK proteins. 
a

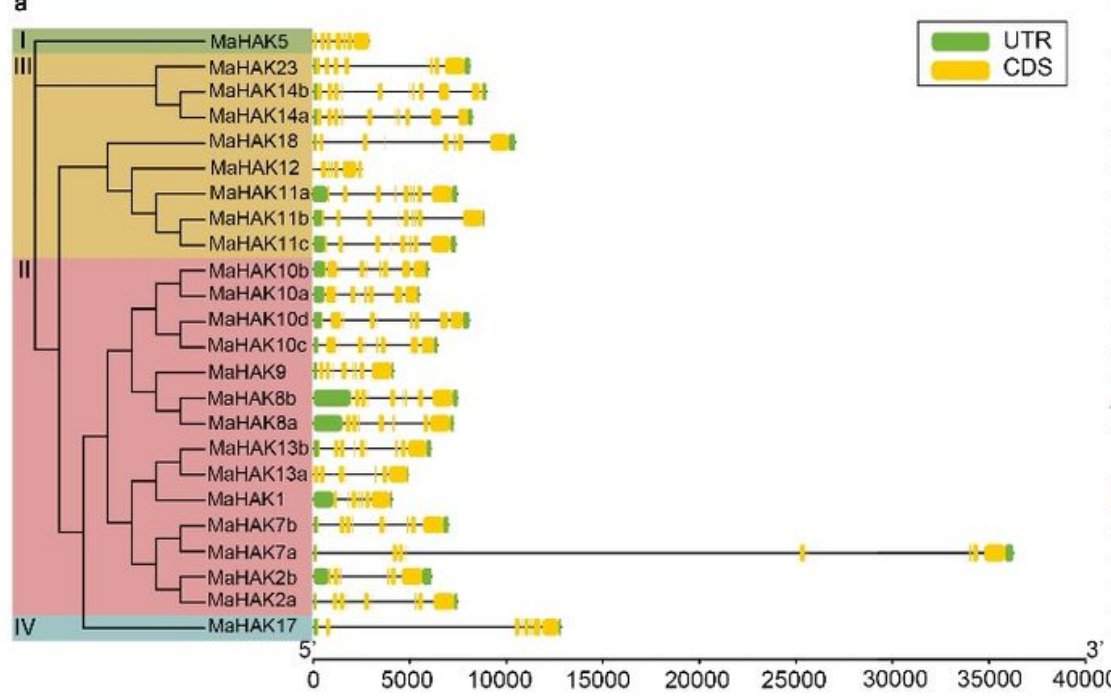

b

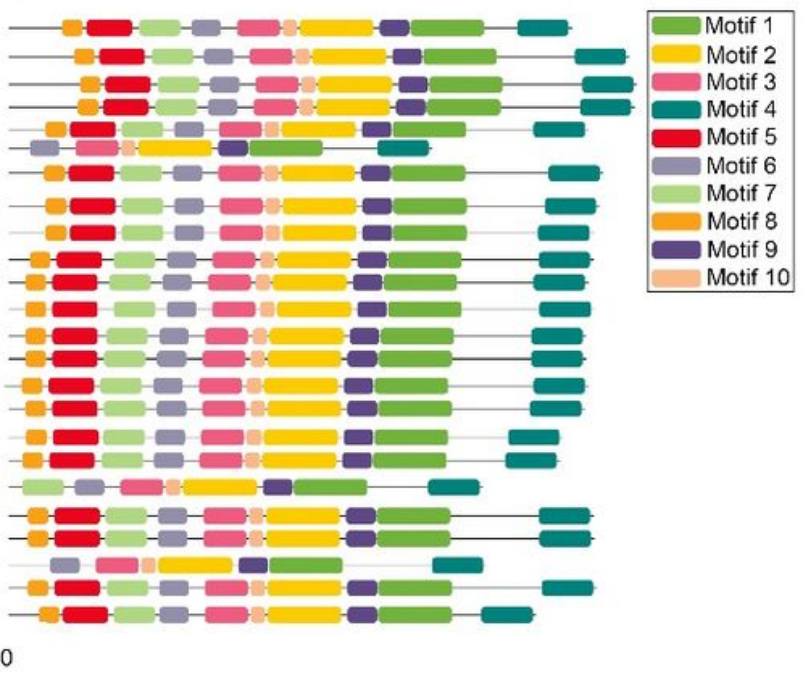

Figure 2

Phylogenetic relationship, gene structures and conserved motifs in MaHAKs. (a) gene structures. MaHAK genes are displayed with the order from the phylogenetic tree constructed based on MaHAK proteins. The green boxes indicate untranslated 5'- and 3'-regions; the yellow boxes and black lines indicate exons and introns, respectively. (b) The motif composition of each of the MaHAK proteins. Ten motifs identified in MaHAK proteins are displayed in different colored boxes. 

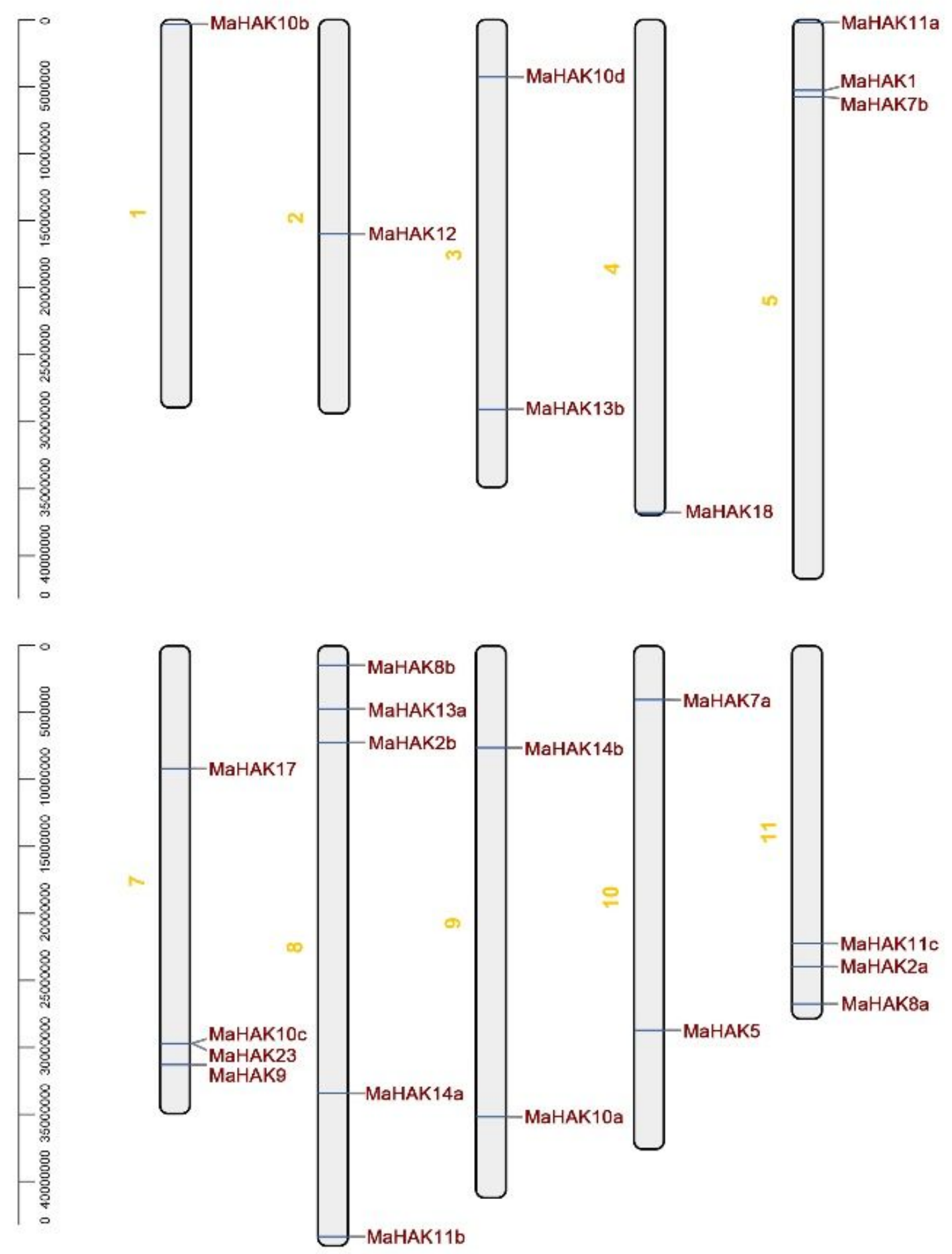

\section{Figure 3}

The chromosome distribution of MaHAK gene. The chromosome numbers are indicated on the right side of each chromosome. The lengths of chromosomes are shown in millions of bases $(\mathrm{Mb})$. 


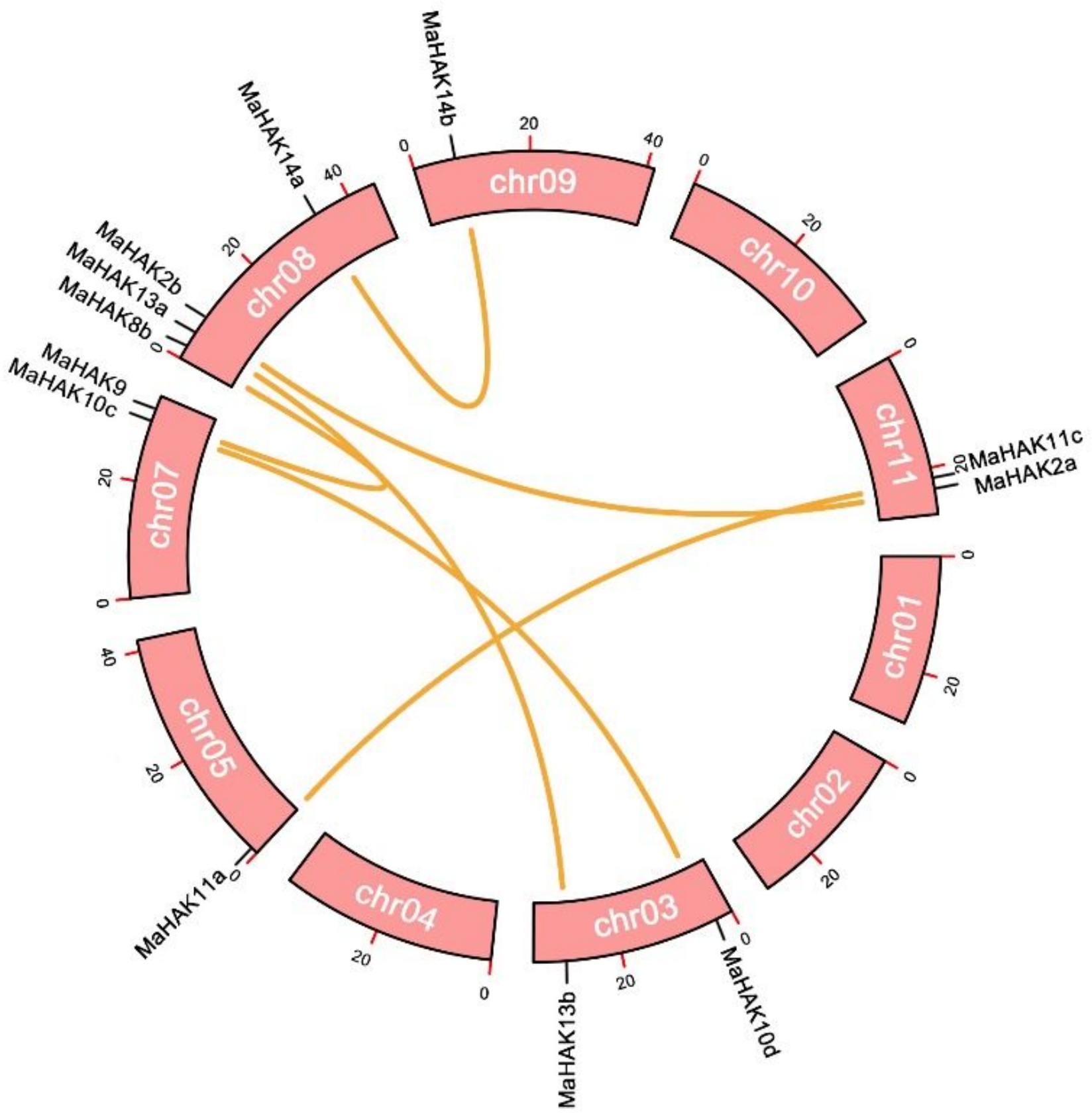

Figure 4

Synteny analysis of the MaHAK gene in the banana genome. The yellow curves indicate duplicated MaHAK gene pairs. 
a

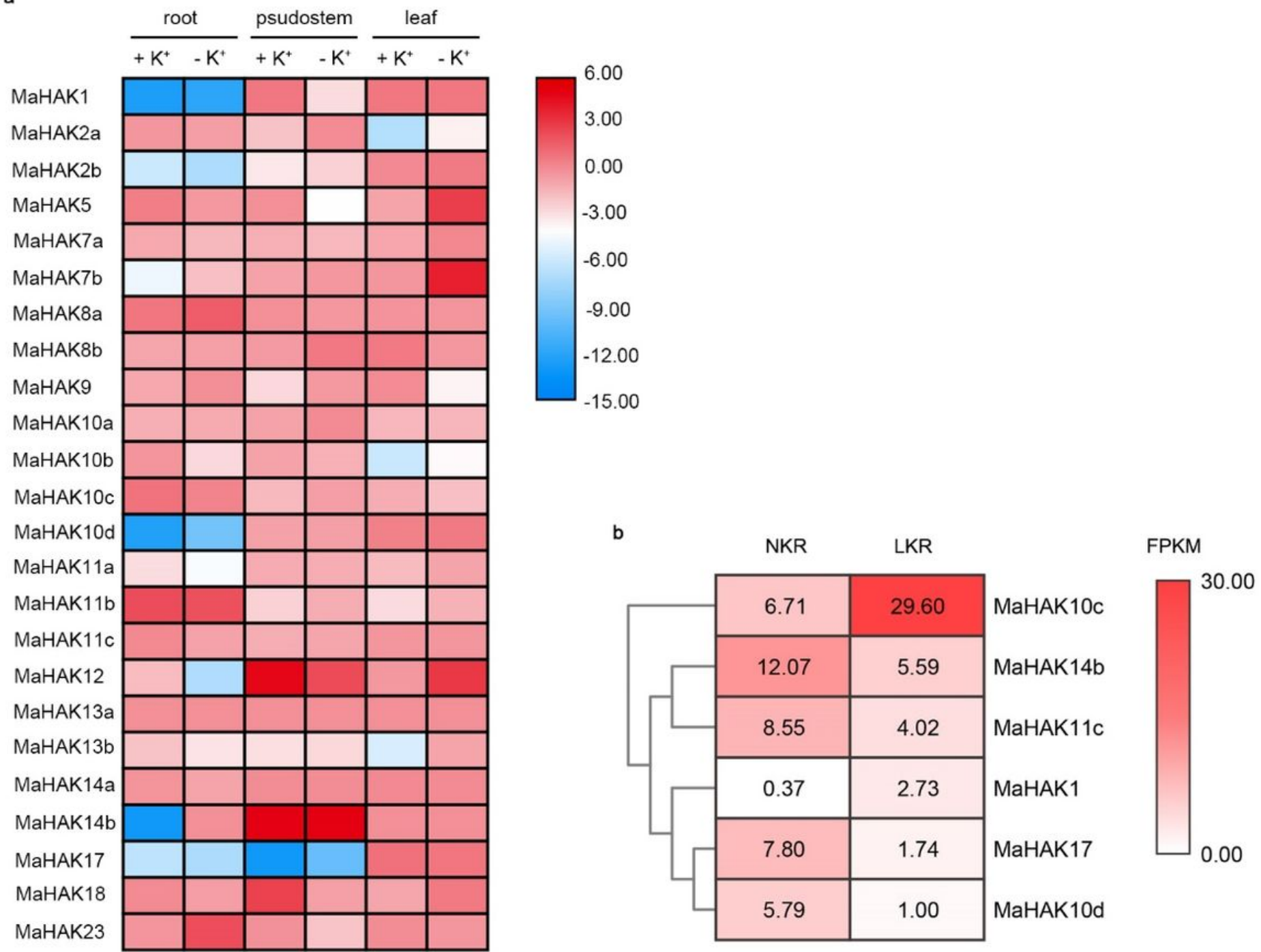

\section{Figure 5}

Expression analysis of MaHAK genes in banana under potassium stress. (a) Expression profiles of MaHAK genes in different tissues for four hours after normal $(+K+)$ and deficient potassium $(-K+)$ treatment. Log2-fold change was used to create the heat map. The color scale represents relative expression levels. (b) The expression pattern of MaHAK genes in roots for $60 \mathrm{~d}$ after different $\mathrm{K}+$ treatments based on FPKM data. 
a
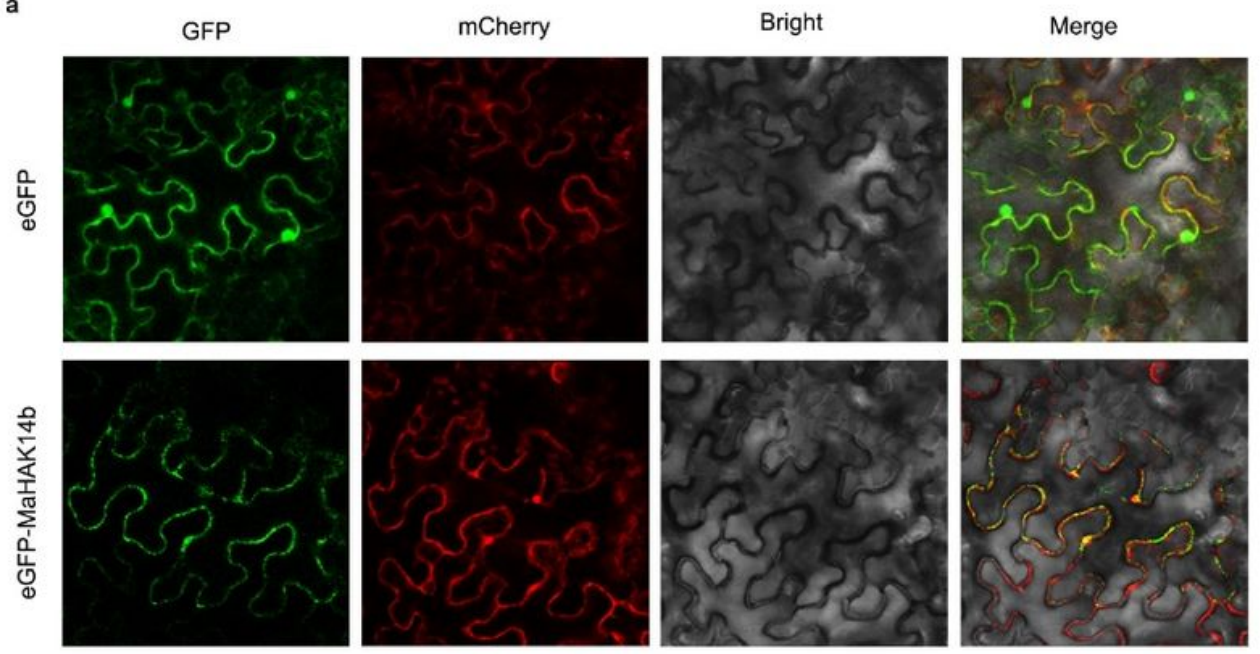

b
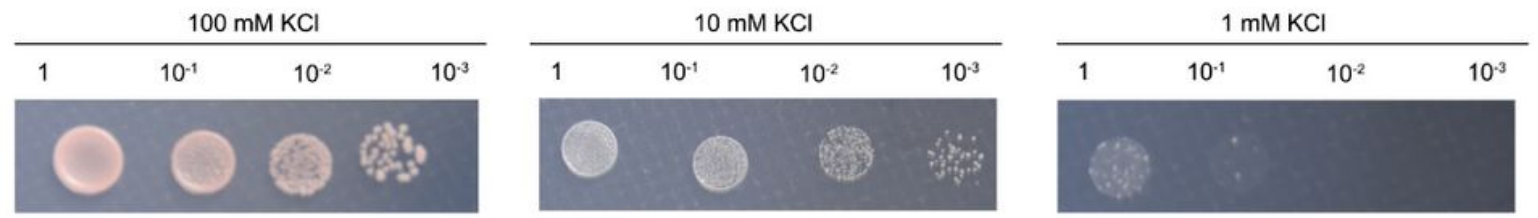

PYES2-MaHAK14b
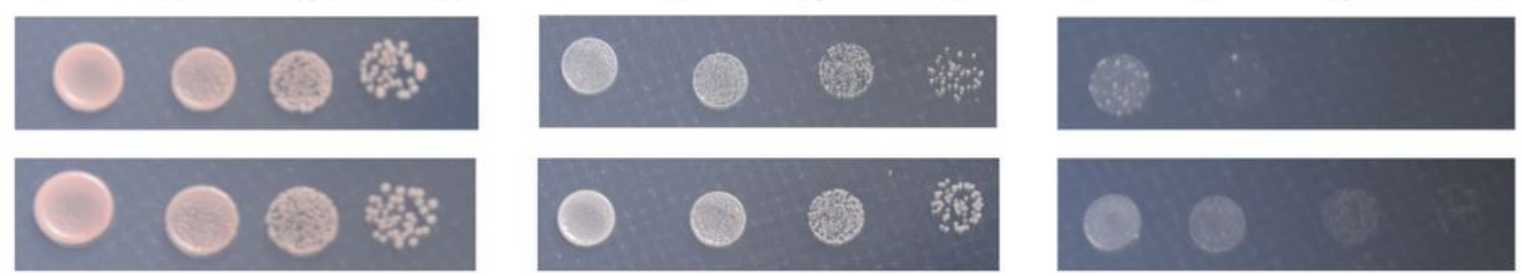

\section{Figure 6}

Functional analyzation of MaHAK14b. (a) Subcellular localization of MaHAK14b. Bar=20 $\mu \mathrm{m}$. (b) The R5421 mutant expressing MaHAK14b grew at low K+ concentrations. 